\title{
A Bayesian Approach for Analyzing the Dynamic Dependence of GDP on the Unemployment Rate in Japan
}

\author{
Koki Kyo and Hideo Noda
}

\begin{abstract}
Real gross domestic product (GDP) and unemployment rate (UR) are basic indicators of business conditions, which are correlated with each other. It is important to understand the dynamic relationship between quarterly GDP and monthly UR. To analyze this dynamic relationship, we propose a Bayesian regression method to estimate the dynamic dependence of a stationary component of GDP on a stationary component of UR. First, we extract the stationary components from the original time series for GDP and UR using a set of state space models. Then, we construct a set of Bayesian regression models, with each model having a time-varying coefficient. As an application of our proposed approach, we analyze the dynamic relationship between the stationary components of GDP and UR in Japan, using the separate URs for men and women. Overall, we find that the movements of UR lead those of GDP by a few months during expansion phases. In contrast, movements of the UR lag those of GDP by a few months during recession phases. Moreover, there is negative correlation between the stationary components of GDP and UR. Such negative correlation is stronger in the recession phase than in the expansion phase. We conclude that the UR can.
\end{abstract}

Index Terms-Bayesian modeling, gross domestic product, unemployment rate, Japanese business cycle.

\section{INTRODUCTION}

Real gross domestic product (GDP) is a basic indicator of business conditions. However, GDP presents shortcomings for business cycle research. A major problem is the timeliness of data because GDP figures are typically published as a quarterly time series [10]. This situation prompted previous research on estimating monthly GDP, to support the need for timely information on business cycles [9], [11], [12].

In this study we propose an approach analyzing the dynamic relationship between a quarterly economic indicator and a monthly economic indicator. In particular, we focus on the relationship between the quarterly GDP and the monthly unemployment rate (UR). The UR is used as a lagging business cycle indicator in both composite indexes and diffusion indexes in Japan. Therefore, we examine the adequacy of the UR as a lagging indicator and potential measure for estimating monthly values of GDP.

Previous studies ([9], [11], [12]) estimating monthly GDP applied regression models in which the coefficients in the models were treated as constant parameters, with the implication that no structural changes occur. However, when

Manuscript received October 9, 2017; revised December 12, 2017.

Koki Kyo is with Obihiro University of Agriculture and Veterinary Medicine, Inada-cho, Obihiro, Hokkaido 080-8555, Japan (e-mail: kyo@obihiro.ac.jp).

Hideo Noda is with Tokyo University of Science, 1-11-2 Fujimi, Chiyoda-ku, Tokyo 102-0071, Japan (e-mail: noda@ rs.tus.ac.jp). the study period spans several decades it is clearly unrealistic implication that no structural changes occur. However, when the study period spans several decades it is clearly unrealistic to assume constant coefficient parameters. Thus, conventional approaches are considered inadequate for analyzing business cycles using long-term time series. [3] developed a Bayesian approach based on vector autoregressive models with time-varying coefficients for analyzing a time series that is nonstationary in covariance. [2] introduced a Bayesian time-varying regression model for dynamic relationship analysis. Recently, these approaches have been applied in [5]-[7]. Here, we use a similar Bayesian dynamic modeling approach to estimate monthly GDP.

The necessary first step in estimating monthly GDP is to analyze the dynamic relationship between quarterly GDP and a monthly economic indicator that can be used as the explanatory variable. In this study, we use UR as the explanatory variable for estimating monthly GDP in Japan. The main problem in estimating monthly GDP lies is determining how to estimate the stationary component of GDP based on the stationary component of UR. Thus, we must first extract stationary components from the original time series for GDP and UR, using a set of state space models. Subsequently, we present a method to analyze the dynamic relationship between the stationary components of GDP and UR using Bayesian dynamic modeling.

There are two important elements of the relationship between GDP and UR, i.e., the lead-lag relationship and the dynamic dependence between these two indicators. The lead-lag relationship is especially useful in analyzing causality between these two indicators. The lead-lag relationship and the dynamic dependence can be expressed by introducing a lag parameter and time-varying coefficients into a set of Bayesian dynamic models. It is also important to explore differences in the relationship between GDP and the URs of different subgroups of the population. Thus, we construct the models using the separate URs for men and women.

The contribution of this study is a new approach for analyzing the dynamic dependence of a quarterly indicator on a monthly indicator. Key features of our approach include clarifying the lead-lag relationship and identifying dynamic effects between these indicators in different phases of the business cycle, and which have not been addressed in earlier studies. Thus, this study may be of particular interest to policy-makers because, in recent years, there has been a growing emphasis on policy evaluation based on rigorous econometric modeling and methods. The proposed approach could also be useful as a tool to verify the assumptions and predicted effects of policies.

Our main results are as follows: We find that movements 
of the UR lead those of GDP by a few months during expansion phases. In contrast, movements of the UR lag those of GDP by a few months during recession phases. Furthermore, there is a negative correlation between the stationary components of GDP and UR. Such negative correlation is stronger in recession phases as compared with expansion phases.

The rest of this paper is organized as follows. In Section II, we introduce a method for estimating a stationary component from the original time series data. In Section III, we show our models and methods of parameter estimation for the proposed approach. An empirical study based on the proposed approach is presented in Section IV. Finally, we discuss our conclusions in Section V.

\section{ESTIMATION OF STATIONARY COMPONENTS}

As mentioned above, the first step in analyzing the relationship between quarterly GDP and monthly UR is the estimation of the stationary components of GDP and UR. Thus, we start by introducing a method for estimating the stationary components from the original time series of GDP and UR.

For the quarterly time series $y_{m}$ of GDP, we consider a set of statistical models as follows:

$$
\begin{aligned}
& y_{m}=t_{m}^{y}+s_{m}^{y}+r_{m}^{y}+w_{m}^{y}, \\
& t_{m}^{y}=2 t_{m-1}^{y}-t_{m-2}^{y}+v_{m 1}^{y}, \\
& s_{m}^{y}=-s_{m-1}^{y}-s_{m-2}^{y}-s_{m-3}^{y}+v_{m 2}^{y}, \\
& r_{m}^{y}=\sum_{j=1}^{p} \alpha_{j} r_{m-j}^{y}+v_{m 3}^{y}(m=1,2, \cdots, M),
\end{aligned}
$$

where $t_{m}^{y}, s_{m}^{y}$, and $r_{m}^{y}$ are, respectively, the trend component, the seasonal component and the stationary component of the time series $y_{m}$. Also, $p$ represents the order of an autoregressive model (AR model) for the stationary components and $\alpha_{1}, \cdots, \alpha_{p}$ are the $\mathrm{AR}$ coefficients. $w_{m}^{y} \sim \mathrm{N}\left(0, \sigma^{2}\right)$ is the observation noise, while $v_{m 3}^{y} \sim \mathrm{N}\left(0, \tau_{1}^{2}\right)$, and $v_{m 3}^{y} \sim \mathrm{N}\left(0, \tau_{3}^{2}\right)$ are system noises for each component model. It is assumed that $w_{m}^{y}, v_{m 1}^{y}, v_{m 2}^{y}$ and $v_{m 3}^{y}$ are independent of one another.

When the model order $p$ and the hyperparameters $\alpha_{1}, \cdots$ $\alpha_{p}, \sigma^{2}, \tau_{1}^{2}, \tau_{2}^{2}$ and $\tau_{3}^{2}$ are given, we can express the models in (1) - (4) by a state space representation. A likelihood function for the hyperparameters is defined using the Kalman filter algorithm, so we can estimate the model order and the hyperparameters using a maximum likelihood method. Then, we can estimate each component in the time series $y_{m}$ using the Kalman filter algorithm, so the estimate for the stationary component $r_{m}^{y}$ of GDP can be obtained (see [4] for detail).

To estimate the stationary component of a monthly time series of UR, we use a set of models similar to that in (1) - (4), as follows:

$$
\begin{aligned}
x_{n} & =t_{n}^{x}+s_{n}^{x}+r_{n}^{x}+w_{n}^{x}, \\
t_{n}^{x} & =2 t_{n-1}^{x}-t_{n-2}^{x}+v_{n 1}^{x}, \\
s_{n}^{x} & =-s_{n-1}^{x}-\cdots-s_{n-11}^{x}+v_{n 2}^{x}, \\
r_{n}^{y} & =\sum_{j=1}^{q} \beta_{j} r_{n-j}^{x}+v_{n 3}^{x}(m=1,2, \cdots, N),
\end{aligned}
$$

where $q$ represents the order of an AR model for the stationary component, $\beta_{1}, \cdots \beta_{q}$ are the AR coefficients. $w_{n}^{x} \sim \mathrm{N}\left(0, \psi^{2}\right)$ is the observation noise, $v_{n 1}^{x} \sim \mathrm{N}\left(0, \eta_{1}^{2}\right)$, $v_{n 2}^{x} \sim \mathrm{N}\left(0, \eta_{2}^{2}\right)$ and $v_{n 3}^{x} \sim \mathrm{N}\left(0, \eta_{3}^{2}\right)$ are system noises. The other quantities correspond to each term in the models in (1) (4). Thus, the model order $q$ and the hyperparameters $\beta_{1}, \cdots$ $\beta_{q}, \psi^{2}, \eta_{1}^{2}, \eta_{2}^{2}$ and $\eta_{3}^{2}$ are estimated using the same algorithm. As a result, the estimate of the stationary component in the time series $r_{n}^{x}$ can be obtained.

\section{METHOD}

\section{A. Models}

To analyze the dynamic relationship between the quarterly GDP and the monthly UR, we propose an adaptation of a modeling approach known as two-mode regression with time-varying coefficients (TMR-TVC).

We classify GDP growth into two states: an upside mode, corresponding to the situation in which the stationary component of GDP is increasing, and a downside mode, corresponding to the situation in which the stationary component is decreasing. We anticipate that the relationship between GDP and UR may differ in these situations, so we use separate models for the two modes.

For the upside mode, the TMR-TVC models are given in the form of a regression model with the time-varying coefficient as follows:

$$
\begin{gathered}
r_{m}^{y}=\sum_{i=1}^{3} \alpha_{3(m-1)+i} r_{3(m-1)+i+L_{1}}^{x}+\varepsilon_{m}^{(1)}, \\
a_{3(m-1)+3}=2 a_{3(m-1)+2}+a_{3(m-1)+1} \\
+e_{3(m-1)+3,}^{(1)} \\
a_{3(m-1)+2}=2 a_{3(m-1)+1}+a_{3(m-1)} \\
+e_{3(m-1)+2,}^{(1)} \\
a_{3(m-1)+1}=2 a_{3(m-1)}+a_{3(m-1)-1} \\
+e_{3(m-1)+1}^{(1)} \\
(m=1,2, \cdots, M),
\end{gathered}
$$

where $r_{m}^{x}$ denotes the estimate of the stationary component in the quarterly time series of GDP, which is obtained from the estimation of models in (1) - (4), and $r_{n}^{x}$ denotes the same for the monthly time series of CS, which is obtained from the estimation of models in (5) - (8). $a_{n}$ is the time-varying coefficient which comprises a monthly time series, and $L_{1}$ denotes a lag. $\varepsilon_{m}^{(1)} \sim \mathrm{N}\left(0, \lambda_{1}^{2}\right)$ is the observation noise and $e_{n}^{(1)} \sim \mathrm{N}\left(0, \phi_{1}^{2}\right)$ is the system noise with $\lambda_{1}^{2}$ and $\phi_{1}^{2}$ being hyperparameters. We assume that $\varepsilon_{m}^{(1)}$ and $e_{n}^{(1)}$ are independent of each other for any values of $m$ and $n$.

The lag $L_{1}$ and the time-varying coefficient $a_{n}$ are two important parameters. From the value of $L_{1}$ we see the lead-lag relationship between GDP and UR; the case where $L_{1}>0$ implies that UR movement lags that of GDP, and the case where $L_{1}<0$ implies that UR movement precedes movement in GDP. Moreover, from the estimate of $a_{n}$ we can analyze the dynamic relationship between GDP and UR.

The models in (9) - (12) are essentially Bayesian linear models, where the model in (9) defines the likelihood, and the models in (10) - (12) form a second order smoothness prior for the time-varying coefficient. So we can estimate the time-varying coefficient with optimal smoothness on $a_{n}$ by controlling the value of $\phi_{1}^{2}$. 
In a similar approach, the TMR-TVC models for the downside mode are given as follows:

$$
\begin{gathered}
r_{m}^{y}=\sum_{i=1}^{3} b_{3(m-1)+i} r_{3(m-1)+i+L_{2}}^{x}+\varepsilon_{m}^{(2)}, \\
b_{3(m-1)+3}=2 b_{3(m-1)+2}+b_{3(m-1)+1} \\
+e_{3(m-1)+3}^{(2)} \\
b_{3(m-1)+2}=2 b_{3(m-1)+1}+b_{3(m-1)} \\
+e_{3(m-1)+2}^{(2)} \\
b_{3(m-1)+1}=2 b_{3(m-1)}+a_{3(m-1)-1} \\
+e_{3(m-1)+1}^{(2)} \\
(m=1,2, \cdots, M)
\end{gathered}
$$

with $L_{2}$ and $b_{n}$ being the lag and the time-varying coefficient. Also, $\varepsilon_{m}^{(2)} \sim \mathrm{N}\left(0, \lambda_{2}^{2}\right)$ is the observation noise and $e_{n}^{(2)} \sim \mathrm{N}\left(0, \phi_{2}^{2}\right)$ is the system noise with $\lambda_{2}^{2}$ and $\phi_{2}^{2}$ are hyperparameters. As in the models in (9) - (12), we assume that $\varepsilon_{m}^{(2)}$ and $e_{n}^{(2)}$ are independent of each other for any values of $m$ and $n$.

Below we show the methods for estimating the hyperparameters in the TMR-TVC models for the upside mode. The methods for the downside mode are similar.

\section{B. Estimation of the Time-Varying Coefficient}

Now, we put

$$
\begin{aligned}
\boldsymbol{z}_{m} & =\left[\begin{array}{l}
a_{3(m-1)+3} \\
a_{3(m-1)+2} \\
a_{3(m-1)+1}
\end{array}\right], \boldsymbol{H}_{m}^{T}=\left[\begin{array}{l}
r_{3(m-1)+3+L_{1}}^{(x)} \\
r_{3(m-1)+2+L_{1}}^{(x)} \\
r_{3(m-1)+1+L_{1}}^{(x)}
\end{array}\right], \\
\boldsymbol{G} & =\left[\begin{array}{ccc}
1 & -2 & 1 \\
0 & 1 & -2 \\
0 & 0 & 1
\end{array}\right]^{-1}=\left[\begin{array}{lll}
1 & 2 & 3 \\
0 & 1 & 2 \\
0 & 0 & 1
\end{array}\right], \\
\boldsymbol{F} & =-\boldsymbol{G}\left[\begin{array}{ccc}
0 & 0 & 0 \\
1 & 0 & 0 \\
-2 & 1 & 0
\end{array}\right]=\left[\begin{array}{lll}
1 & 2 & 3 \\
0 & 1 & 2 \\
0 & 0 & 1
\end{array}\right], \\
\boldsymbol{e}_{m} & =\left[\begin{array}{l}
e_{3(m-1)+3}^{(x)} \\
e_{3(m-1)+2}^{(x)} \\
e_{3(m-1)+1}^{(x)}
\end{array}\right], \boldsymbol{Q}=\mathrm{E}\left\{\boldsymbol{e}_{\boldsymbol{m}} \boldsymbol{e}_{m}^{T}\right\}=\phi_{1}^{2} \boldsymbol{I}_{3}
\end{aligned}
$$

With $\boldsymbol{I}_{3}$ denoting a 3 -th identity matrix. Then, the models in (9) - (12) can be expressed by the following state space model:

$$
\begin{aligned}
\boldsymbol{z}_{m} & =\boldsymbol{F} \boldsymbol{z}_{m-1}+\boldsymbol{G} \boldsymbol{e}_{m}, \\
r_{m}^{y} & =\boldsymbol{H}_{m} \boldsymbol{z}_{m}+\varepsilon_{m}^{(1)} .
\end{aligned}
$$

In the state space model comprising (17) and (18), the time-varying coefficient $a_{n}$ is included in the state vector $\boldsymbol{z}_{m}$ Therefore, the estimate for $a_{n}$ can be obtained from the estimate of $\boldsymbol{z}_{m}$. Moreover, the parameters, $\lambda_{1}^{2}$ and $\phi_{1}^{2}$, labeled hyperparameters, can be estimated using the maximum likelihood method.

Let $\boldsymbol{z}_{0}$ denote the initial value of the state and $Y_{1}^{(k)}$ denote a set of estimates for $r_{m}^{y}$ up to time point $k$, where $k$ denotes a quarter. Assume that $\boldsymbol{z}_{0} \sim \mathrm{N}\left(\boldsymbol{z}_{0 \mid 0}, \boldsymbol{C}_{0 \mid 0}\right)$. Because the distribution $f\left(\mathbf{z}_{m} \mid Y_{1}^{(k)}\right)$ for the state $\boldsymbol{z}_{m}$ conditional on $Y_{1}^{(k)}$ is Gaussian, it is only necessary to obtain the mean $\boldsymbol{z}_{m \mid k}$ and the covariance matrix $\boldsymbol{C}_{m \mid k}$ of $\boldsymbol{z}_{m}$ with respect to $f\left(\mathbf{z}_{m} \mid Y_{1}^{(k)}\right)$.

Given the values of $L_{1}, \lambda_{1}^{2}$ and $\phi_{1}^{2}$, the initial distribution $\mathrm{N}\left(\boldsymbol{z}_{0 \mid 0}, \boldsymbol{C}_{0 \mid 0}\right)$, and a set of estimates for $r_{m}^{x}$ up to time point $M$, the means and covariance matrices in the predictive distribution and filter distribution for the state $\boldsymbol{z}_{m}$ can be obtained using the Kalman filter for $m=1,2, \cdots, M$ (see [4] for example):

\section{[Prediction]}

$$
\begin{aligned}
\boldsymbol{z}_{m \mid m-1} & =\boldsymbol{F} \mathbf{z}_{m-1 \mid m-1}, \\
\boldsymbol{C}_{m \mid m-1} & =\boldsymbol{F} \boldsymbol{C}_{m-1 \mid m-1} \boldsymbol{F}^{\mathbf{t}}+\boldsymbol{G} \boldsymbol{Q} \boldsymbol{G}^{\mathbf{t}} .
\end{aligned}
$$

\section{[Filter-A]}

$$
\begin{aligned}
& \boldsymbol{K}_{m}=\boldsymbol{C}_{m-1 \mid m-1} \boldsymbol{H}_{m}^{\mathbf{t}}\left(\boldsymbol{H}_{m} \boldsymbol{C}_{m-1 \mid m-1} \boldsymbol{H}_{\boldsymbol{m}}^{\mathbf{t}}+\lambda_{1}^{2}\right)^{-1}, \\
& \boldsymbol{z}_{m \mid m}=\boldsymbol{z}_{m \mid m-1}+\boldsymbol{K}_{m}\left(r_{m}^{y}-\boldsymbol{H}_{m} \boldsymbol{z}_{m \mid m-1}\right), \\
& \boldsymbol{C}_{m \mid m}=\left(\boldsymbol{I}_{3}-\boldsymbol{K}_{m} \boldsymbol{H}_{m}\right) \boldsymbol{C}_{m \mid m-1} .
\end{aligned}
$$

[Filter-A]

$$
\begin{aligned}
\boldsymbol{z}_{m \mid m} & =\boldsymbol{z}_{m \mid m-1}, \\
\boldsymbol{C}_{m \mid m} & =\boldsymbol{C}_{m \mid m-1} .
\end{aligned}
$$

Note that, for each value of $m$, when the time point $m$ is in an upside period we use the step Filter-A; otherwise Filter-B is applied.

Based on the results of the Kalman filter, we can obtain the estimate for $\boldsymbol{z}_{m}$ using the fixed-interval smoothing for $m=$ $M-1, M-2, \cdots, 1$ as follows:

\section{[Fixed-Interval Smoothing]}

$$
\begin{aligned}
& \boldsymbol{A}_{m}=\boldsymbol{C}_{m \mid m} \boldsymbol{F}^{\mathbf{t}} \boldsymbol{C}_{m+1 \mid m}^{-1}, \\
& \boldsymbol{z}_{m \mid M}=\boldsymbol{z}_{m \mid m}+\boldsymbol{A}_{m}\left(\mathbf{z}_{m+1 \mid M}-\boldsymbol{z}_{m+1 \mid m}\right), \\
& \boldsymbol{C}_{m \mid M}=\boldsymbol{C}_{m \mid m}+\boldsymbol{A}_{m}\left(\boldsymbol{C}_{m+1 \mid M}-\boldsymbol{C}_{m+1 \mid m}\right) \boldsymbol{A}_{m}^{\mathbf{t}} .
\end{aligned}
$$

Then, the posterior distribution of $\boldsymbol{z}_{m}$ can be given by $\boldsymbol{z}_{m \mid M}$ and $\boldsymbol{C}_{m \mid M}$. Subsequently, the estimate for the time-varying coefficient $a_{n}$ can be obtained because the state space model described by (17) and (18) incorporates $a_{n}$ in the state vector $\boldsymbol{z}_{m}$.

\section{Estimation of the Constant Parameters}

Given the time series data $Y_{1}^{(M)}=\left\{r_{1}^{y}, r_{2}^{y}, \cdots, r_{M}^{y}\right\}$ and the corresponding time series data $\left\{r_{1}^{x}, r_{2}^{x}, \cdots, r_{3 M}^{x}\right\}$, a likelihood function for the hyperparameters $\lambda_{1}^{2}$ and $\phi_{1}^{2}$ and the parameter $L_{1}$ is given by:

$$
f\left(Y_{1}^{(M)} \mid \lambda_{1}^{2}, \phi_{1}^{2}, L_{1}\right)=\prod_{m=1}^{M} f_{m}\left(r_{m}^{y} \mid \lambda_{1}^{2}, \phi_{1}^{2}, L_{1}\right),
$$

where $f_{m}\left(r_{m}^{y} \mid \lambda_{1}^{2}, \phi_{1}^{2}, L_{1}\right)$ is the density function of $r_{m}^{y}$. As demonstrated by [4], when using the Kalman filter, the density function $f_{m}\left(r_{m}^{y} \mid \lambda_{1}^{2}, \phi_{1}^{2}, L_{1}\right)$ is a normal density given by: 


$$
\begin{gathered}
f_{m}\left(r_{m}^{y} \mid \lambda_{1}^{2}, \phi_{1}^{2}, L_{1}\right)=\frac{1}{\sqrt{2 \pi w_{m} \mid m-1}} \\
\times \exp \left\{-\frac{\left(r_{m}^{y}-\hat{r}_{m-1}^{y}\right)^{2}}{2 w_{m \mid m-1}}\right\},
\end{gathered}
$$

where $\hat{r}_{m-1}^{y}$ is the one-step ahead prediction for $r_{m}^{y}$ and $w_{m \mid m-1}$ is the variance of the predictive error, respectively:

$$
\begin{aligned}
& \hat{r}_{m-1}^{y}=\boldsymbol{H}_{m} \boldsymbol{z}_{m \mid m-1}, \\
& w_{m \mid m-1}=\boldsymbol{H}_{m} \boldsymbol{C}_{m-1 \mid m-1} \boldsymbol{H}_{\boldsymbol{m}}^{\mathbf{t}}+\lambda_{1}^{2} .
\end{aligned}
$$

Moreover, for a fixed value of $L_{1}$, the estimates of the hyperparameters can be obtained using the maximum likelihood method, i.e., we can estimate the hyperparameters by maximizing $f\left(Y_{1}^{(M)} \mid \lambda_{1}^{2}, \phi_{1}^{2}, L_{1}\right)$ in (19) together with (20) In practice, when we substitute the new $\lambda_{1}^{2}=1$ into the above Kalman filter algorithm, the estimate $\hat{\lambda}_{1}^{2}$ for $\lambda_{1}^{2}$ is obtained analytically by:

$$
\hat{\lambda}_{1}^{2}=\frac{1}{M} \sum_{m=1}^{M} \frac{\left(r_{m}^{y}-\hat{r}_{m \mid m-1}^{y}\right)^{2}}{w_{m \mid m-1}} .
$$

So, the estimate $\hat{\phi}_{1}^{2}$ for $\phi_{1}^{2}$ can be obtained by maximizing $f\left(Y_{1}^{(M)} \mid \lambda_{1}^{2}, \phi_{1}^{2}, L_{1}\right)$ under the use of (21).

Information about the value of lag $L_{1}$, which is important for analyzing the lead-lag relationship between GDP and UR, can obtained from the maximum value of the likelihood function. For a given value of the lag $L_{1}$, the maximum likelihood is given as $f\left(Y_{1}^{(M)} \mid \hat{\lambda}_{1}^{2}, \hat{\phi}_{1}^{2}, L_{1}\right)$, then for a set $\left\{L_{1}^{(1)}, L_{1}^{(1)}+1, \cdots, L_{1}^{(2)}-1, L_{1}^{(2)}\right\}$ of $L_{1}$ we can calculate the relative likelihood by:

$$
\begin{gathered}
R\left(L_{1}\right)=\frac{f\left(Y_{1}^{(M)} \mid \hat{\lambda}_{1}^{2}, \hat{\phi}_{1}^{2}, L_{1}\right)}{\sum_{j=L_{1}^{(1)}}^{L_{1}^{(2)}} f\left(Y_{1}^{(M)} \mid \hat{\lambda}_{1}^{2}, \hat{\phi}_{1}^{2}, j\right)} \\
\left(L_{1}=L_{1}^{(1)}, L_{1}^{(1)}+1, \cdots, L_{1}^{(2)}-1, L_{1}^{(2)}\right)
\end{gathered}
$$

So, we can analyze the lead-lag relationship between GDP and UR from the distribution of the relative likelihood on $L_{1}$.

\section{EMPIRICAL STUDY}

To analyze GDP behavior as a reference cycle, we use UR data for men and women in Japan. The data for real GDP were obtained from the Cabinet Office, Government of Japan. The UR data were obtained from the website of the Ministry of Internal Affairs and Communications, Japan.

Fig. 1 shows the quarterly time series of real GDP in Japan for the period 1980Q1 - 2005Q4.

Fig. 2 and Fig. 3 are line graphs showing the monthly time series of UR for men and women, respectively, during 1978.1 - 2007.12.

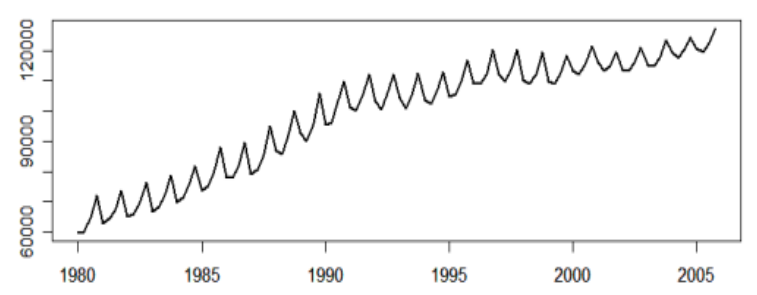

Fig. 1. Time series data of real GDP in Japan (1980Q1 - 2005Q4).

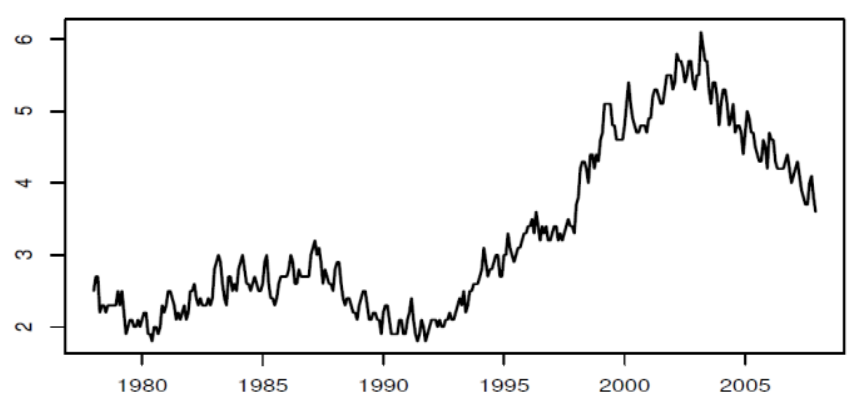

Fig. 2. Time series data of UR for men in Japan (1978.1 - 2007.12).

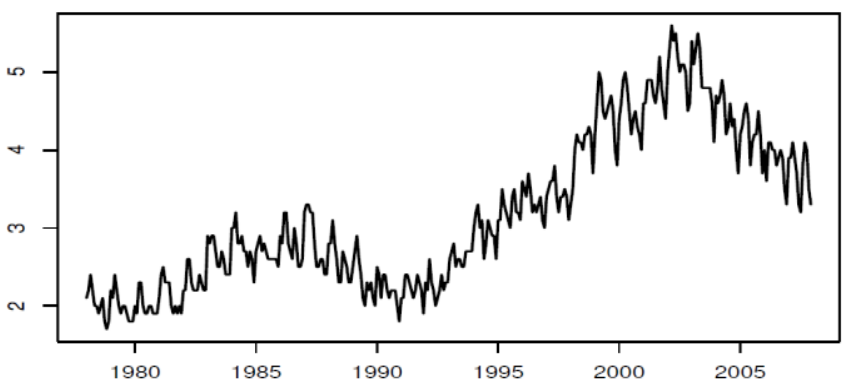

Fig. 3. Time series data of UR for women in Japan (1978.1 - 2007.12).

Note that GDP is measured in billions of Japanese Yen, while UR is measured in percent.

For simplicity in parameter estimation, we adjust the scale for the GDP time series. Specifically, letting $y_{m}^{*}$ denote the original data for GDP, we adjust the associated scale by:

$$
y_{m}=100 \times \frac{y_{m}^{*}}{y_{1}^{*}} \quad(m=1,2, \cdots) .
$$

Also, because the unit of the data $x_{n}^{*}$ for each time series of UR is percent, we logarithmically transform the UR time series as follows:

$$
x_{n}=\log \left(x_{n}^{*}\right) \quad(n=1,2, \cdots)
$$

In the analysis below, we use the scale-adjusted time series $y_{m}$ as the data for GDP and use the logarithmic transformed time series $x_{n}$ as the data for UR.

To estimate the stationary component in GDP, we compute the likelihoods for the models in (1) - (4) with $p=1,2,3,4$. The maximum likelihood is obtained when $p=1$. So we use models with $p=1$ as a set of best models in our data analysis. To estimate the stationary components of UR for men and women, we compute the likelihoods for the models in (5) - (8) with $q=1,2, \cdots, 6$. The maximum values of likelihood are 
obtained for the models for men and women with $q=5$ and $q=3$, respectively. Thus, in the data analysis we use the models for UR of men with $q=5$ and that for UR of women with $q=3$.

Fig. 4 shows the estimate for the stationary component of GDP.

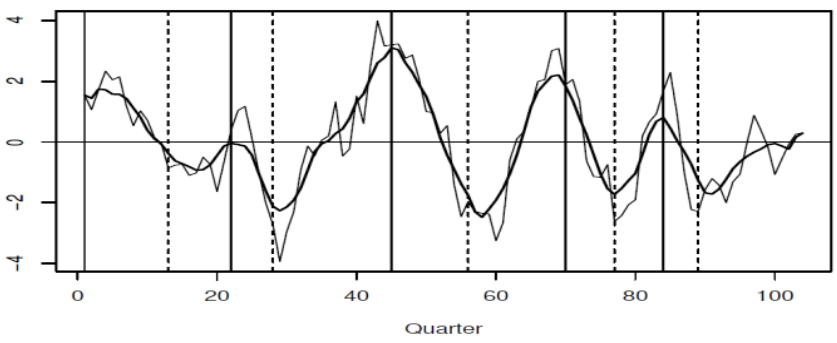

Fig. 4. Time series for the estimation of the stationary components of real GDP. The vertical lines indicate turning points in the business cycle (the solid and broken lines indicate peaks and troughs, respectively).

The thin line expresses the value for the original estimate and the thick line shows that for a 7-quarter moving average. The vertical lines indicate inflections of the business cycle, where the solid and broken lines indicate peaks and troughs, respectively. It can be seen from Figure 4 that fluctuation in the stationary component in GDP correlates closely with business cycles in Japan.

Fig. 5 and 6 show the estimates for the stationary components of the URs for men and women, respectively.

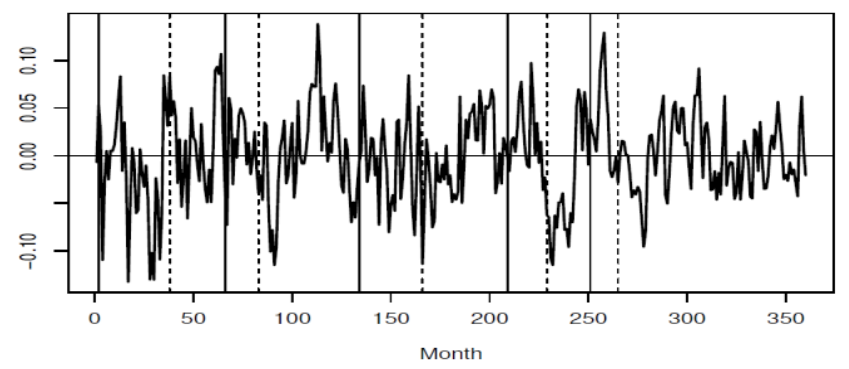

Fig. 5. Time series for the estimation of the stationary component of the UR for men. The vertical lines indicate inflections of the business cycle (the solid and broken lines indicate peaks and troughs, respectively).

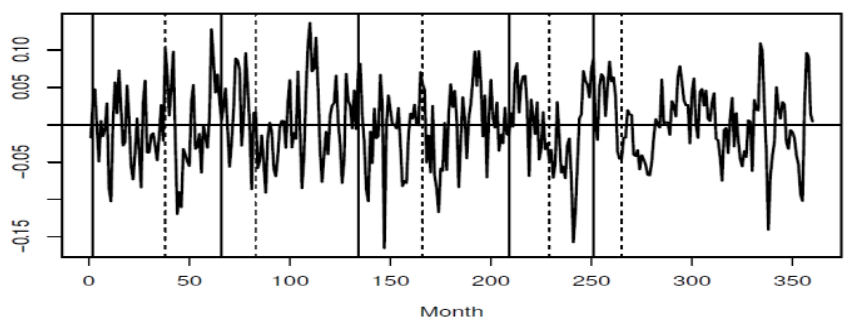

Fig. 6. Time series for the estimation of the stationary component of the UR for women. The vertical lines indicate inflections of the business cycle (the solid and broken lines indicate peaks and troughs, respectively).

As in Fig. 4, the vertical lines indicate inflections of the business cycle, where the solid and broken lines indicate peaks and troughs, respectively. However, it is difficult to see, simply through visual examination of the figures, a clear relationship between business cycles and the fluctuations of the stationary components of these URs.

Fig. 7 shows the relative likelihood distribution of the lag, measured in months, between -24 and 24 in the model for UR for men. (a) for upside mode model

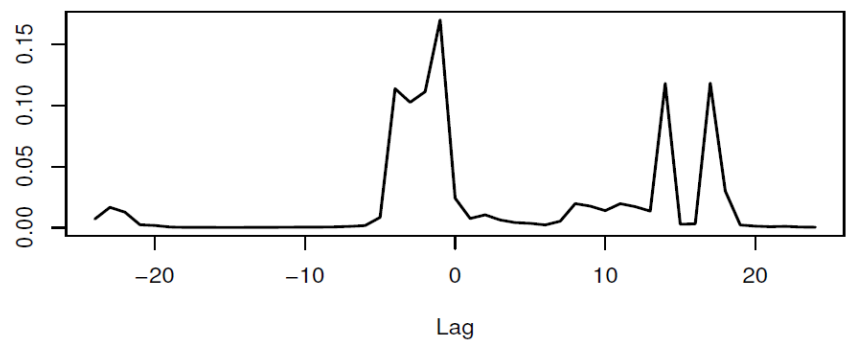

(b) for downside model model

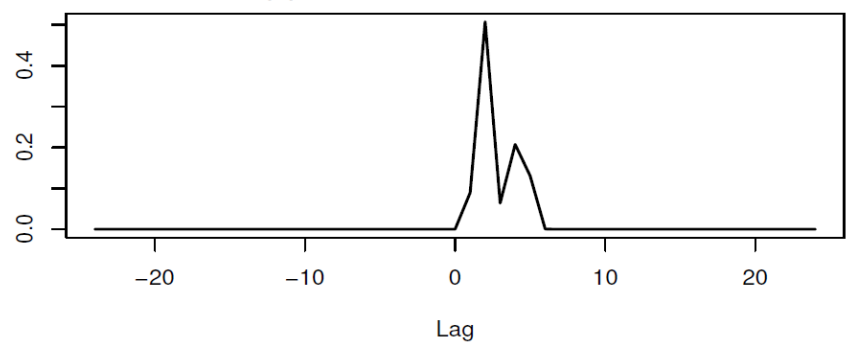

Fig. 7. Relative likelihood distribution on the lag for UR for men.

From Fig. 7, we see some interesting characteristics. First, many peaks exist in the upside mode model. Specifically, there are left and right peaks, approximately corresponding to two months ahead and 17 (or 14) months later. Simply put, the lead-lag relationship between GDP and UR for men is complicated during expansion phases. Movements in UR for men sometimes lead GDP by two months while lagging GDP by 17 months at other times. In other words, sometimes the UR for men leads business expansion by about 2 months so that it could be interpreted as a cause of business cycle. At other times, UR for men lags business expansion by about one and a half years, hence it could be considered an effect of economic growth. However, the results for the downside mode model show a peak in the relative likelihood at approximately two months lag.

Fig. 8 shows time series of estimates for the time-varying coefficients in the models of UR for men.

(a) for upside mode model

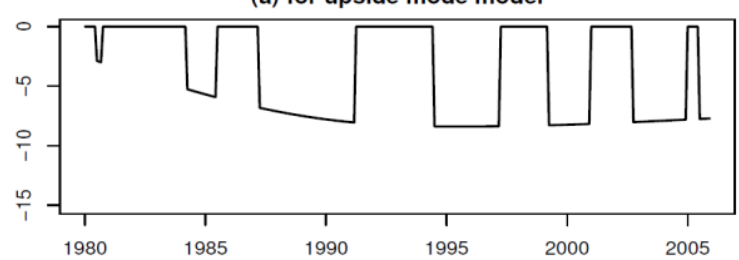

(b) for downside mode model

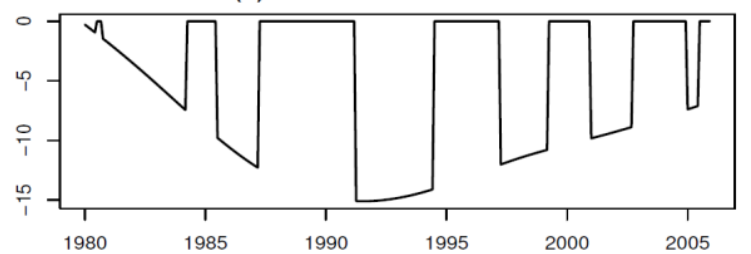

Fig. 8. Time series of the time-varying coefficient for the UR for men.

In the upside mode model, $L_{1}=-2$ and in the downside mode model, $L_{2}=2$. From the estimation results, we can see that during both the upside mode and the downside mode, the estimates for the time-varying coefficient take negative values in many periods. We note some distinctive characteristics of these models. For the upside mode, the 
time-varying coefficients indicate a relatively regular pattern after the late 1980s. In the downside mode, the absolute values of the coefficient are relatively large during the early 1990s. After the late 1990s, however, the absolute values of the coefficient become progressively smaller. Further, the absolute values of the coefficient in the downside mode are larger than those of the coefficient in the upside mode. These results imply that there is negative correlation between the stationary components in GDP and UR for men. In particular, the negative correlation is stronger in the recession phase.

Fig. 9 shows the relative likelihood distribution on the lags between -24 and 24 months in the models of UR for women.

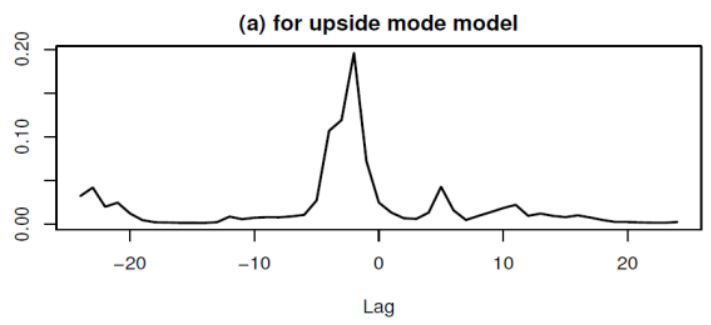

(b) for downside model model

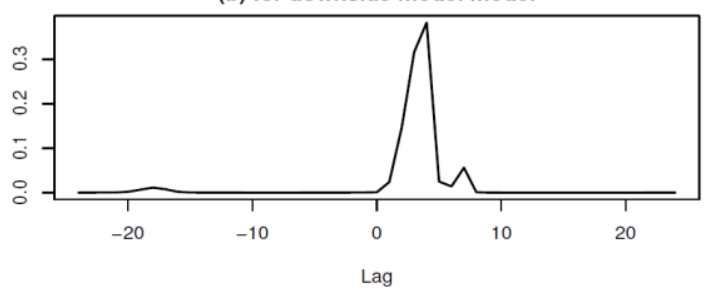

Fig. 9. Relative likelihood distribution on the lag for UR for women.

(a) for upside mode model

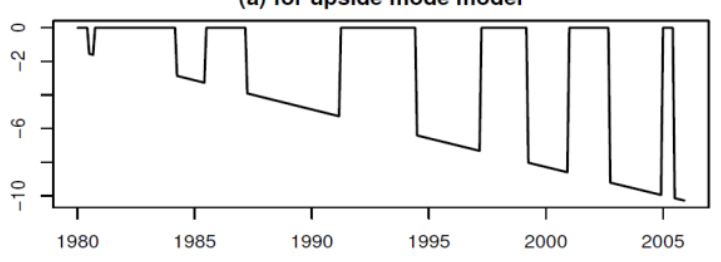

(b) for downside mode model

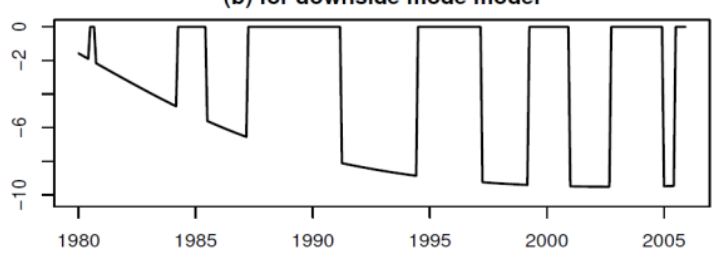

Fig. 10. Time series of the time-varying coefficients for UR for women.

The results shown in Fig. 9 are more easily interpreted than those in Fig. 7. For the upside mode model, the relative likelihood indicates a peak value occurring two months ahead. For the downside mode model, the relative likelihood indicates the peak value occurring four months behind. In other words, during expansion phases, movements of UR for women lead GDP movements by two months. Conversely, during recession phases, GDP movements lead UR movements by four months. Because the peaks of relative likelihood are observed in the neighborhood of lag 0 for the models of both upside and downside modes, we might interpret that movements of UR for women correlate closely with movements of GDP.

Fig. 10 shows time series of estimates for the time-varying coefficients in the models of UR for women, in which the panel of the upside mode model (a) is for $L_{1}=-2$ and the panel of the downside mode model (b) is for $L_{2}=4$.

Similarly to the results shown in Figure 8, the estimates for the time-varying coefficient for both the upside mode and the downside mode take negative values in many periods. Also, the absolute values of the coefficient in the downside mode are larger than those in the upside mode. However, it can be seen that the absolute values of the coefficient for both modes become larger in recent years. This implies that the negative correlation between the stationary components in GDP and UR for women has been strengthening in recent years.

Conventionally, the relationships between UR and GDP have been studied from the perspective of Okun's law (see, for example, [1] and [8]). In general, however, empirical studies of Okun's law ignore considerations of the phase of the business cycle and the time-varying effects of UR on GDP. Our Bayesian modeling and empirical work offer new findings on the relationships between UR and GDP.

\section{CONCLUSION}

We proposed a Bayesian dynamic linear modeling method for analyzing the dynamic relationship between a quarterly economic indicator and a monthly economic indicator. We considered GDP as an example for the quarterly indicator and unemployment rate (UR) as that for the monthly indicator.

The proposed method can be used to estimate monthly GDP. For monthly GDP estimation, we focused on the dynamic relationship between GDP and UR in Japan, and presented a set of Bayesian regression models for the stationary component in GDP based on the use of the stationary component in UR. We classified GDP growth into two states: an upside mode, corresponding to the situation in which the stationary component of GDP is increasing, and a downside mode, corresponding to the situation in which the stationary component is decreasing. To express the dynamic relationship between GDP and UR for the upside mode and the downside mode, the Bayesian regression models are constructed in the form of two-mode regression with time-varying coefficients (TMR-TVC).

It should be noted that there are two important parameters in the TMR-TVC models: One is the time lag between GDP and UR. The other is the time-varying coefficient. We can determine the lead-lag relationship between GDP and UR from the value of the lag and thus analyze the causality between these two indicators. Moreover, from the estimate of the time-varying coefficient, we can analyze the dynamic relationship between GDP and UR.

A key contribution of the present study is the development of a new method for analyzing the dynamic dependence of quarterly GDP on monthly UR. An important feature of this method involves clarifying the lead-lag relationship and dynamic effects between GDP and UR in different phases of the business cycle. These elements were not addressed by earlier studies therefore this approach can offer greater insight into the relationships between different economic measures than previous research. Specifically, this study is likely to be of particular interest to policy-makers, because emphasis on policy evaluation based on rigorous econometric modeling and methods has grown in recent years. The proposed approach can also be useful as a tool to verify 
policy assumptions or to forecast the effects of policies. In other words, this study also makes a methodological contribution to economic studies.

The usefulness of this approach has been demonstrated by the empirical example. Using Japanese time series data and our new method, we empirically examined the dynamic relationship between the stationary components of GDP and UR for men and women, respectively. The main results were as follows: (1) The lead-lag relationship between GDP and UR for men during an expansion phase is not easily interpreted. (2) There is a negative correlation between the stationary components of GDP and UR for men. In our dataset, the negative correlation was stronger in the recession phase after the bubble economy period in Japan. (3) The dynamic relationship between GDP and UR for women is more straightforward than that for men. More specifically, movements of the UR lead those of GDP by a few months during the expansion phase, while movements of the UR lag those of GDP by a few months during a recession phase.

From the more comprehensive perspective enabled by our method, we therefore conclude that it is reasonable to include the UR in a lagging indicator during a recession phase. However, it may be inappropriate as part of a lagging indicator during an expansion phase.

\section{ACKNOWLEDGMENT}

This work is supported in part by a Grant-in-Aid for Scientific Research (C) (16K03591) from the Japan Society for the Promotion of Science. We thank Deborah Soule, DBA from Edanz Group (www.edanzediting.com/ac) for editing a draft of this manuscript.

\section{REFERENCES}

[1] K. Hamada and Y. Kurosaka, "The relationship between production and unemployment in Japan: Okun's law in comparative perspective," European Economic Review, vol. 25, no. 1, pp. 71-91, June 1984.

[2] X-Q. Jiang and K. Kyo, "A bayesian method for the dynamic regression analysis," Transactions of the Institute of Systems, Control and Information Engineers, vol. 8, no. 1, pp. 8-16, January 1995.

[3] X-Q. Jiang, K. Kyo, and G. Kitagawa, "A time varying coefficient vector AR modeling of nonstationary covariance time series," Signal Processing, vol. 33, no. 3, pp. 315-331, September 1993.

[4] G. Kitagawa, Introduction to Time Series Modeling, CRC Press, 2010.

[5] K. Kyo and H. Noda, "A new algorithm for estimating the parameters in seasonal adjustment models with a cyclical component," ICIC Express Letters: An International Journal of Research and Surveys, vol. 5 , no. 5 pp. 1731-1737, May 2011
[6] K. Kyo and H. Noda, "Bayesian analysis of the dynamic relationship between oil price fluctuations and industrial production performance in Japan," Information: An International Interdisciplinary Journal, vol. 16, no. 7A: 4639-4660, July 2013.

[7] K. Kyo and H. Noda, "Dynamic effects of oil price fluctuations on business cycle and unemployment rate in Japan," International Journal of Innovation, Management and Technology, vol. 6, no. 6, pp. 374-377, December 2015.

[8] Y. Kurosaka, "Okun's law and employment adjustment," Japanese Economy, vol. 39, no. 2, pp. 87-107, Summer 2012.

[9] H. Liu and S. G. Hall, "Creating high-frequency national accounts with state-space modelling: a Monte Carlo experiment," Journal of Forecasting, vol. 20, no. 6, pp. 441-449, September 2001

[10] R. S. Mariano and Y. Murasawa, "A new coincident index of business cycles based on monthly and quarterly series," Journal of Applied Econometrics, vol. 18, no. 4, pp. 427-443, July-August 2003.

[11] R. S. Mariano and Y. Murasawa, "A coincident index, common factors, and monthly real GDP," Oxford Bulletin of Economics and Statistics, vol. 72, no. 1, pp. 27-46, February 2010.

[12] B. Seong, S. K. Ahn, and P. Zadrozny, "Estimation of vector error correction models with mixed-frequency data," Journal of Time Series Analysis, vol. 34, no. 2, pp. 194-205, March 2013.

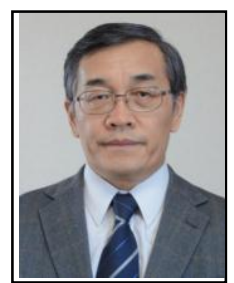

Koki Kyo was born in Shenyang, China, on Sept. 27, 1957. He received his BE degree and ME degree in management engineering from Northeastern University, China, in 1982 and 1984 respectively, and $\mathrm{PhD}$ degree in statistical science from the Graduate University for Advanced Studies, Japan, in 1993. He is a professor of statistics at Obihiro University of Agriculture and Veterinary Medicine. His current research interests include bayesian statistical modeling, estimation and identification of dynamic systems and Bayesian methods of economic analysis. Prof. Kyo is a member of the Japan Statistical Society, a member of the Institute of Systems, Control and Information Engineers, and many others.

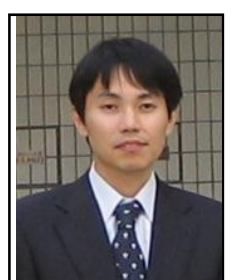

Hideo Noda was born in Saga, Japan, on Aug. 26, 1972. He received Ph.D. in economics from Kyushu University, Japan, in 2004. He has been an associate professor at Tokyo University of Science, Japan, since 2013. His work has appeared in Economic Issues, Empirical Economics Letters, Journal of Economic Research, and many others. His current research interests include economic growth, business cycles and global environmental problems. Dr. Noda is a member of the Japanese Economic Association. 